\title{
Smart-phone and medical app use amongst Irish medical students: a survey of use and attitudes
}

\author{
G Browne*, D O'Reilly, C Waters, O Tummon, D Devitt, B Stewart, P O'Connor \\ From International Conference for Healthcare and Medical Students (ICHAMS) 2013 \\ Dublin, Ireland. 11-12 October 2013
}

\section{Background}

Studies in the UK and Canada reveal high smart-phone ownership rates with the majority of students viewing these devices as very useful with regards to their clinical education. Worryingly, low awareness basic privacy and security measures appears common amongst medical students. In Ireland, little is known regarding smartphone app ownership and use. This study sampled Irish undergraduate medical students at a single site.

\section{Methods}

A 31-item questionnaire was developed by the primary researcher following a preliminary literature review and subsequently underwent peer review. The questionnaire was distributed by means of a paper survey. Non-probability convenience sampling was conducted at educational sessions at a single site to students of all years of a medical undergraduate curriculum as per ethics approval. Collected data was analysed using SPSS Statistics 20. The internal consistency of the questionnaire as measured by Cronbach's alpha was high $(\alpha=0.951)$.

\section{Results}

The survey response rate was $34.8 \%$ (317/909) with $80.8 \%$ (256/317) of respondents owning a smart-phone. A greater percentage of preclinical students, 83.4\% (151/181) owned smart-phones as compared to older students, of which $77.3 \%$ owned such a device $(105 / 29)$. More clinical students $(78.1 \%)$ used medical apps as compared to preclinical students (57\%). The two most popular brands were Apple and Samsung devices. Of those who owned a smart-phone, 65.6\% (168/256) reported using medicallyrelated apps. Students used apps predominately to aid their study. While $69.9 \%(179 / 256)$ of respondents trusted the information provided by the medical apps they used,

$$
\text { Department of Medicine, National University of Ireland, Western Road, }
$$$$
\text { Galway, Ireland }
$$ 